\title{
Adsorption geometry determination of single molecules by atomic force microscopy
}

\author{
Bruno Schuler, ${ }^{1, *}$ Wei Liu, ${ }^{2}$ Alexandre Tkatchenko, ${ }^{2}$ Nikolaj Moll, ${ }^{1}$ \\ Gerhard Meyer, ${ }^{1}$ Anish Mistry, ${ }^{3}$ David Fox,${ }^{3}$ and Leo Gross ${ }^{1}$ \\ ${ }^{1}$ IBM Research - Zurich, Säumerstrasse 4, 8803 Rüschlikon, Switzerland \\ ${ }^{2}$ Fritz-Haber-Institut der Max-Planck-Gesellschaft, Faradayweg 4-6, 14195 Berlin, Germany \\ ${ }^{3}$ University of Warwick, Gibbet Hill, CV34 Warwick, UK
}

(Dated: July 24, 2013)

\begin{abstract}
We measured the adsorption geometry of single molecules with intra-molecular resolution using noncontact atomic force microscopy (NC-AFM) with functionalized tips. The lateral adsorption position was determined with atomic resolution, adsorption height differences with a precision of $3 \mathrm{pm}$, and tilts of the molecular plane within $0.2^{\circ}$. The method was applied to five $\pi$-conjugated molecules, including three molecules from the olympicene family, adsorbed on $\mathrm{Cu}(111)$. For the olympicenes, we found that the substitution of a single atom leads to strong variations of the adsorption height, as predicted by state-of-the-art density-functional theory including van der Waals interactions with collective substrate response effects $\left(\mathrm{DFT}+\mathrm{vdW} \mathrm{Wurf}^{\text {sur }}\right)$.
\end{abstract}

PACS numbers: 68.37.Ps, 34.20.Gj, 68.35.-p, 68.43.-h

In noncontact atomic force microscopy (NC-AFM) the crucial factors affecting the image contrast are the chemical interaction between probe and sample [1], the tip termination [2-4] and the adsorbate geometry [5]. For organic molecules on metal substrates, the adsorption geometry (adsorption site, height, tilt) is intimately linked to the electronic properties of the adsorbate and the interaction between adsorbate and substrate [6]. In other words, the adsorption geometry is a direct indicator of the adsorbate-substrate-interaction. The adsorption height of molecules above the substrate is traditionally measured using the X-ray standing wave method (XSW) $[7,8]$. While XSW allows to determine the adsorption height with high precision and chemical sensitivity, it does not (yet) provide information about the lateral adsorption position or tilt angle. Because XSW values are averaged over large ensembles, individual molecules are not distinguished. In contrast, using scanning probe microscopy, molecules are treated individually and therefore the molecular adsorption geometry can be measured as a function of molecular conformation [5] or the adsorption site [9]. The adsorption site of single adsorbates can be determined by scanning tunneling microscopy (STM) using marker atoms $[10,11]$ or inelastic electron tunneling spectroscopy [12] or by directly resolving substrate and adsorbate by AFM $[5,13,14]$. However, to date, adsorption heights could not be quantified by scanning probe microscopy.

In this letter we present a novel experimental approach to extract the molecular adsorption geometry in full detail by AFM and compare our results to density-functional theory (DFT) calculations. First, the method of determining heights is exemplified for pentacene and diindeno[1,2,3-cd:1',2',3'-lm]perylene (DIP) and the role of our tip termination, carbon monoxide $(\mathrm{CO})$ and $\mathrm{Xe}$, is discussed. Thereafter, we apply the method to three molecules of the olympicene family, $6 \mathrm{H}-$ benzo[cd]pyrene (olympicene), benzo[cd]pyrene (radical), $6 \mathrm{H}$-benzo[cd]pyren-6-one (ketone), which differ in their chemical structure only by one atom. Finally, adsorption sites of the olympicenes are determined by atomically resolving the substrate and the adsorbed molecule in one image. The molecules are investigated on $\mathrm{Cu}(111)$.

Our measurements were performed with a combined STM/AFM using a qPlus tuning fork sensor [15] operated in the frequency modulation mode [16] (oscillation amplitude $=0.5 \AA$ ) under ultrahigh vacuum $\left(p \approx 10^{-11} \mathrm{mbar}\right)$ and low temperature $(T \approx 5 \mathrm{~K})$ conditions. For the tip functionalization we grew two monolayer thick $\mathrm{NaCl}$ islands on the $\mathrm{Cu}(111)$ single crystal $[\mathrm{NaCl}(2 \mathrm{ML}) / \mathrm{Cu}(111)]$ and adsorbed $\mathrm{CO}$ and $\mathrm{Xe}$ on the sample, which were vertically manipulated to functionalize the tip (for details see Supplemental Material (SM) [17] or Ref. [4]). The molecules to be studied were subsequently evaporated onto the cold sample.

In Fig. 1 (a) the scheme of our method for measuring adsorption heights is illustrated. To access the molecular adsorption height and tilt we determine for different lateral positions $(x, y)$ the height $z^{*}(x, y)$ where the frequency shift $\Delta f(x, y, z)[18]$ is minimal: $z^{*}(x, y)=$ $\arg \min _{z}\{\Delta f(x, y, z)\}$ with respect to the correspondent substrate value. To obtain a $z^{*}$ map, individual $\Delta f(z)$ spectra were recorded with variable tip approach on a $2 \mathrm{D}$ grid above the molecule (for details see [19]). To compare the molecular adsorption height, tilt and bending between experiment and theory we fit a geometry model and extract the adsorption height at a certain reference point, tilt angle and bending from this fitted model.

In general, the $z^{*}(x, y)$ values will differ from real adsorption heights $z_{\text {ad }}(x, y)$ [see Fig. $1(\mathrm{~h})$ ]. The observed offset, $z_{\text {off }}^{*}=z_{\text {ad }}(x, y)-z^{*}(x, y)$, which depends on the tip termination, originates from the chemically inequivalent species being probed for the calibration: $\mathrm{Cu}$ on the substrate and $\mathrm{C}$ on the molecule. Moreover, this offset is 
(a)

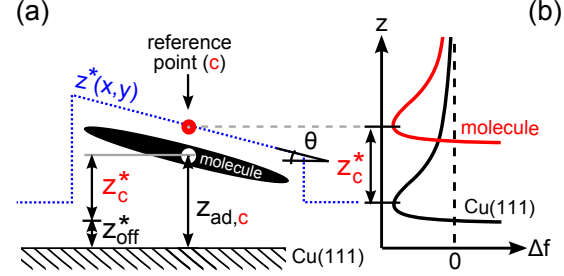

(b)



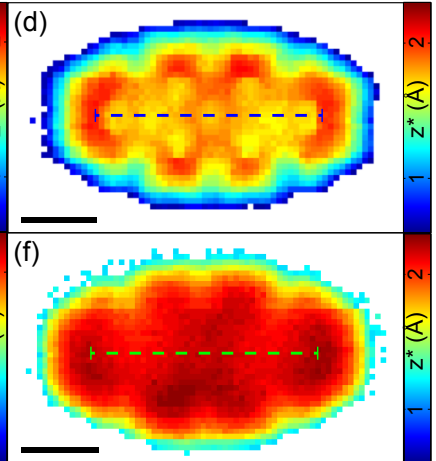

(h)

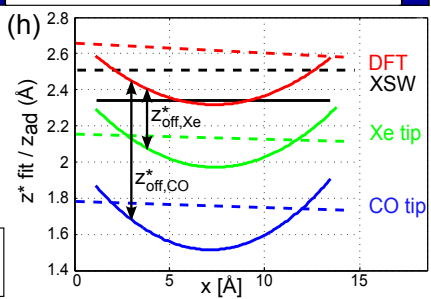

(g)

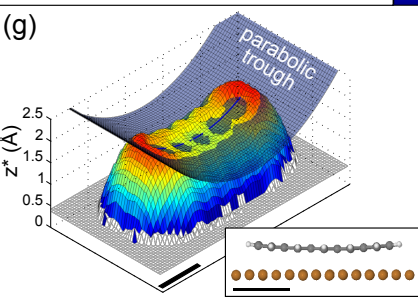

(c)

(e)



FIG. 1. (a) Schematic of the adsorption height determination. (b) Pentacene and DIP model. (c-f) The tip height $z^{*}$ at minimal $\Delta f$ on pentacene $(\mathrm{c}, \mathrm{e})$ and $\operatorname{DIP}(\mathrm{d}, \mathrm{f})$ on $\mathrm{Cu}(111)$ with $\mathrm{CO}(\mathrm{c}, \mathrm{d})$ an $\mathrm{Xe}(\mathrm{e}, \mathrm{f})$ terminated tip. $z^{*}(x, y)$ is extracted from a $3 \mathrm{D} \Delta f$ map and given with respect to the $z^{*}$ value on $\mathrm{Cu}(111)$ with the respective tip. White points mark spectra where the $\Delta f$ minimum was not reached during data acquisition. (g) 3D representation of (c) with a fitted parabolic trough model indicated in blue. The inset depicts the calculated geometry for pentacene on $\mathrm{Cu}(111)$ using DFT+vdW ${ }^{\text {surf }}$. (h) Line profiles of the fitted parabolic trough for pentacene (solid lines) and plane for DIP (dashed lines) for the measurements in (c)-(f) and calculated geometries using DFT+vdW ${ }^{\text {surf }}$ along the molecules' long axis [see lines in (c)-(f)]. The black lines mark the experimental XSW values (no lateral information). Note that there is a tip dependent offset $z_{\text {off,CO }}^{*}, z_{\text {off,Xe }}^{*}$ between the calculated and AFM measured values. Scale bars: $5 \AA$.

sensitive to the sample bias and macroscopic tip shape. Therefore, only adsorption height differences can be determined even if the tip does not change during the measurement. However, to facilitate an absolute adsorption height determination the offset can be gauged by $z^{*}$ measurements on a molecule with known adsorption height (done here) or by calculating the minimal frequency shift on the substrate and molecule with an appropriate tip model. Furthermore, the bias dependence of $z_{\text {off }}^{*}$ could be reduced by measurements at compensated bias (local contact potential difference, LCPD). However, the LCPD depends on the lateral and vertical tip position

[20], which makes it difficult to account for.

In the following, $z^{*}(x, y)$ maps recorded on similar molecules (in extend and composition) with identical CO or Xe tips are compared at zero bias. First, pentacene and DIP shown in Fig. 1 (b) are investigated. The fact that absolute adsorption height values are known for pentacene and DIP from XSW measurements and theory allows us to link the measured $z^{*}$ values to absolute height values. Maps of $z^{*}$ with CO tips [Fig. 1 (c,d)] are atomically corrugated, whereas Xe tips [Fig. 1 (e,f)] give a smoother contrast being predominantly susceptible to the collective molecular geometry. With both tips we observed increased $z^{*}$ above the ends of pentacene with respect to its molecular center. In Fig. 1 (g) a 3D representation of Fig. 1 (c) is depicted, in good agreement with a parabolic trough model, overlaid in blue. Superimposed to the parabolic behavior along the molecule's long axis there is also a small tilt along the short axis observed (see SM [17]).

Since XSW measurements can only provide averaged values for adsorption heights, DFT calculations were performed to gain site-specific adsorption height information. These calculations are challenging due to the interplay of Pauli repulsion, covalent interactions, electron transfer processes and van der Waals (vdW) interactions [21]. The DFT+vdW ${ }^{\text {surf }}$ method [22], which is a synergetic combination of the DFT+vdW method [23] for inter-molecular interactions with the Lifshitz-ZarembaKohn theory for the non-local Coulomb screening within the bulk, predicts adsorption heights of organic molecules on coinage surfaces with an accuracy of $0.1 \AA[24,25]$. In the following the $\mathrm{DFT}+\mathrm{vdW} \mathrm{Wurf}^{\text {surf }}$ method is applied to our measured systems by using Perdew-Burke-Ernzerhof [26] for the exchange-correlation functional. In the inset of Fig. 1 (g) the calculated geometry of pentacene on $\mathrm{Cu}(111)$ is displayed. As for the measurements, a parabolic trough for pentacene and plane for DIP is fitted to the relaxed molecule structures, which is presented in Fig. 1 (h). For pentacene and DIP the adsorption height difference between both molecules and the curvature of pentacene are in good agreement with the calculated adsorption geometry (see Tab. 1 in the SM [17] for all adsorption heights and angles) and XSW measurements $[24,27]$. By comparison to the DFT data we find that $z_{\text {off }}^{*}$ in the AFM measurements was $\approx 0.8 \AA$ for the $\mathrm{CO}$ and $\approx 0.4 \AA$ for the Xe tip shown in Fig. 1. Although the results obtained with $\mathrm{CO}$ and Xe tips match the calculations comparably well, we will restrict ourself in the following to measurements of $z^{*}$ with Xe tips to avoid possible influence from $\mathrm{CO}$ bending, which has been reported to affect the $\Delta f$ contrast $[28,29]$. Moreover, the smooth $z^{*}$ contrast of the Xe tip makes $z_{\text {off }}^{*}$ independent of the specific molecule site that is probed.

Now we will apply the method introduced above to another set of $\pi$-conjugated molecules, which we will call olympicenes. The olympicenes are three molecules 
formed of five carbon rings. They differentiate from each other by the atom(s) bound to the carbon at the edge of the central carbon ring [see insets of Fig. 2 (a)]. In the case of olympicene there is a $s p^{3}$ hybridized carbon atom forming a $\mathrm{C}-\mathrm{H}_{2}$ moiety. For the radical, which is created on the surface by dehydrogenation [30] of the olympicene by applying a voltage pulse of $1.6 \mathrm{~V}$ at $10 \mathrm{pA}$, the carbon atom is $s p^{2}$ hybridized having a single hydrogen bound to the carbon $(\mathrm{C}-\mathrm{H})$. The ketone has a carbonyl group $(\mathrm{C}=\mathrm{O})$ at that position.

The calculations show very distinct adsorption height differences between the olympicenes. Olympicene is physisorbed (greatest adsorption height), ketone is in an intermediate regime between physi- and chemisorption, whereas radical is chemisorbed [31] (smallest adsorption height). Despite noticeable differences in the adsorption distances, we find that the olympicenes have very similar adsorption energies. This effect will be analyzed in detail in Ref. [32]. The olympicenes adsorb in a planar but tilted geometry. To compare the adsorption heights of this tilted geometry, the central carbon atom serves as reference point. The $z^{*}$ and $z_{\mathrm{ad}}$ value (of the fitted model) at this reference point are denoted as $z_{\mathrm{c}}^{*}$ and $z_{\mathrm{ad}, \mathrm{c}}$, respectively.

The measured heights $z^{*}$ for ketone shown in Fig. 3 are discussed in detail. Like in the case of DIP we assume from the calculation the structure to lie in a plane described by $z_{i}^{*}=f\left(x_{i}, y_{i} ; z_{\mathrm{c}}^{*}, \theta\right)$, where the $z^{*}$ value of the spectrum $i$ at the position $\left(x_{i}, y_{i}\right)$ is given by $z_{\mathrm{c}}^{*}$ and $\theta$ describing the height offset and tilt angle, respectively. The plane is fitted by the least mean squares error method to those spectra of the $3 \mathrm{D} \Delta f$ map that are lying within the circumference of the calculated structure model. The structure model was manually placed to the $\Delta f$ contrast in a constant height slab $\Delta f(x, y, z=$ const) [see inset in Fig. 3 (a)]. From the fitted plane, the tilt angle $\theta$ and $z_{\mathrm{c}}^{*}$ are extracted. In Fig. 3 (b) the calculated geometry of ketone is shown. The measured and calculated tilt angles are in good agreement. By comparing $z^{*}$ to the DFT calculations of the olympicenes we find for Xe tips that $z_{\text {off }}^{*}=0.4 \pm 0.2 \AA$, i.e. similar values as for pentacene and DIP.

In Fig. 2 the different olympicenes are compared. In Fig. 2 (a) a histogram of the residuals of $z^{*}$ with respect to the least-squares fitted plane plus the corresponding $z_{\mathrm{c}}^{*}$ value are plotted. At the central carbon atom the adsorption height differences of the olympicenes can be identified. The normally distributed residuals of ketone and radical imply the appropriate choice of our geometry model (plane). In contrast, the residuals of olympicene are less well described by the Gaussian because we observe a small bending of the molecule perpendicular to its symmetry axis. This small bending, which is also observed in the calculations, makes the structure not perfectly described by our geometry model.

To estimate the error of the fitting parameters $z_{\mathrm{c}}^{*}$ and


FIG. 2. (Color online) (a) Probability distribution of $z^{*}$ recorded with a Xe tip around the least-squares fitted plane with tilt angle $\theta$ and height $z_{\mathrm{c}}^{*}$ for the olympicenes. The black curves correspond to fitted Gaussian line shapes. The insets illustrate the molecule structures of the olympicenes, where the positions of other hydrogens have been omitted for clarity. (b) Contour plot of the root-mean-squares error $Q$ between $z^{*}$ and a plane with $z_{\mathrm{c}}^{*}$ and $\theta$ as free parameters. The contours are shown for multiples of the standard deviation of the least squares fitted plane $\sigma$. The blue error bars mark the standard error of the parameters. (a)

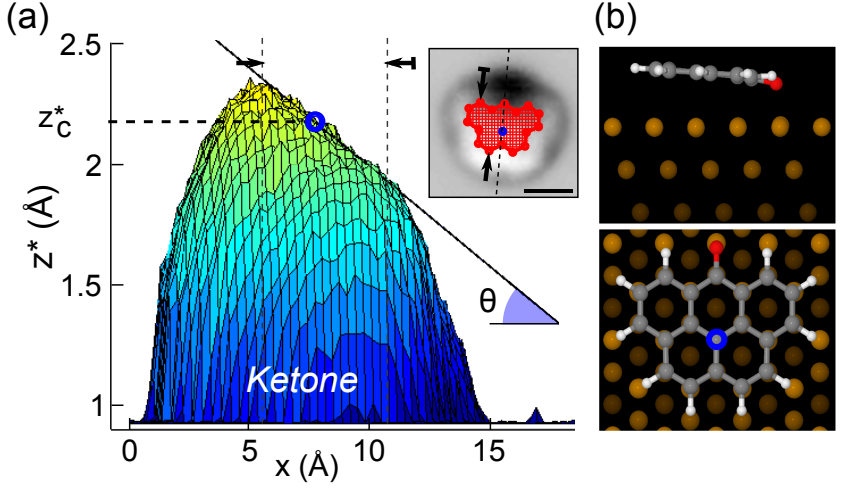

FIG. 3. (Color online) (a) Side-view on the $z^{*}$ surface recorded with a Xe tip along the symmetry axis (inset dashed line) of ketone with the least-squares fitted plane (black line) indicated. The inset depicts a constant height slab $\Delta f(x, y, z=\mathrm{const})$ of the $3 \mathrm{D} \Delta f$ map indicating the spectra (total number $N=257$ ) within the circumference of the calculated structure model used for fitting the plane. The black arrows mark the dimension of the carbon framework in direction of its symmetry axis. The $z^{*}$ value from the fitted plane at the central carbon position (blue circle) is $z_{\mathrm{c}}^{*}=2.21 \pm 0.01 \AA$. The experimental adsorption angle is $\theta=4.9 \pm 0.1^{\circ}$. (b) Side- and top-view on the calculated geometry of ketone on $\mathrm{Cu}(111)$ using $\mathrm{DFT}+\mathrm{vdW} \mathrm{Wrrf}^{\text {sur] }}$. Scale bar: $5 \AA$. 
TABLE I. Adsorption heights of the olympicenes from $z^{*}$ and $\mathrm{DFT}+\mathrm{vdW}$ surf with $z_{\mathrm{ad}, \mathrm{c}}=z_{\mathrm{c}}^{*}+z_{\text {off }}^{*}$.

\begin{tabular}{lcrrr}
\hline \hline & \multicolumn{2}{c}{ AFM } & \multicolumn{2}{c}{ DFT } \\
& $z_{\mathrm{c}}^{*}$ & $\theta$ & $z_{\mathrm{ad}, \mathrm{c}}$ & $\theta$ \\
\hline olympicene & $2.58 \pm 0.03 \AA$ & $-1.1 \pm 0.2^{\circ}$ & $2.85 \AA$ & $-0.4^{\circ}$ \\
ketone & $2.21 \pm 0.01 \AA$ & $4.9 \pm 0.1^{\circ}$ & $2.66 \AA$ & $5.9^{\circ}$ \\
radical & $2.08 \pm 0.03 \AA$ & $3.3 \pm 0.1^{\circ}$ & $2.62 \AA$ & $3.4^{\circ}$ \\
\hline \hline
\end{tabular}

$\theta$, the root-mean-square errors $Q$ are plotted in Fig. 2 (b) as a function of both fitting parameters with isolines at multiples of the standard deviation $\sigma$ of the least squares fit. $Q$ is given by

$$
Q=\sqrt{\frac{1}{N} \sum_{i=1}^{N}\left(z_{i}^{*}-f\left(x_{i}, y_{i} ; z_{\mathrm{c}}^{*}, \theta\right)\right)^{2}}
$$

where $N$ is the number of considered spectra. The error bars indicating the standard error of the parameters are defined by the contour line with the value $Q=\sigma \sqrt{1+1 / N}$. The relative adsorption height difference and tilt angle between the olympicenes can clearly be distinguished. As for ketone, the measured tilt angles for olympicene and radical compare very well with the calculated tilt angles (see Tab. I). Note that the high accuracy of $z_{\mathrm{c}}^{*}$ and $\theta$ is a consequence of the exponentially decaying Pauli repulsion $[3,33]$ and the reproducibility of $z^{*}$ from the different $\Delta f$-distance spectra during one measurement.

The adsorption sites were determined by a method that we call adjusted constant height AFM. To atomically resolve substrate and molecule, a CO terminated tip is scanned in constant height mode at a smaller height distant from the molecule and at a greater height above the molecule. By extracting the stacking sequence at a step edge, $h c p$ and $f c c$ hollow sites can be differentiated globally on each terrace, for our single crystal [see Fig. 4 (a)]. Note that for the (111) face, the atom positions can be identified due to symmetry reasons. With the $\mathrm{CO}$ tip we find that the $\mathrm{Cu}$ atom sites are more attractive (darker) than the atomic interspace in the operated distance regime. Olympicene and ketone were found to adsorb either on $h c p 30^{\circ}$ or $f c c 30^{\circ}$ sites (measured three times per molecule and site) with respect to their carbon ring centers visible in Fig. 4 (b,d). The $30^{\circ}$ describes the azimuthal angle between the close-packed directions of the (111) face to the direction connecting two opposing atoms in the carbon rings of the molecule. The radical on the other hand was only observed on $f c c 30^{\circ}$ sites (measured five times). This could be explained by the larger substrate coupling observed by the smaller adsorption height. Of course other possible adsorption sites, though unlikely, can not be completely excluded. The simplicity, rapidity and accuracy of the introduced method without requiring marker atoms is very beneficial. Note that we


FIG. 4. (Color online) Adjusted constant height AFM images with $\mathrm{CO}$ functionalized tips (tip height $z$ changes are marked by red lines). Tip heights are given with respect to an STM conductance set point of $G=10 \mathrm{pS}$. The $\Delta f$-scale is optimized on each part of the image (brighter means a less negative $\Delta f$ value). Crossings of black continuous lines mark $\mathrm{Cu}$ atom positions (the grid was adapted to each image). (a) The atom positions of both $\mathrm{Cu}$ layers at a step edge (grid of upper $\mathrm{Cu}$ layer is continued as dashed grid on lower layer) determine the $f c c$ sites (triangles pointing up, marked blue) and $h c p$ sites (triangles pointing down, marked red). The adsorption sites are given with respect to the centers of the $\mathrm{C}_{6}$ rings. (b) Olympicene on $h c p 30^{\circ}$ site (also $f c c 30^{\circ}$ observed). (c) Radical on $f c c 30^{\circ}$ site (exclusive adsorption site). (d) Ketone on $f c c 30^{\circ}$ (also $h c p 30^{\circ}$ observed). Scale bars: $5 \AA$.

can correlate the individual molecular adsorption geometry with the adsorption site. For the shown molecules we observed no influence of the adsorption site on the adsorption height or tilt within our measurement error.

We measured the adsorption site, height and tilt of single molecules by AFM using $\mathrm{CO}$ and Xe functionalized tips. The adsorption height is the sum of a tip dependent offset $z_{\mathrm{off}}^{*}$ and $z^{*}$ that reflects the molecular adsorption geometry. The demonstrated small statistical error of $3 \mathrm{pm}$ for $z^{*}$ facilitates a high sensitivity to inter- and intra-molecular differences in adsorption heights. Therefore we could determine the differences in adsorption height and tilt for the olympicenes. Furthermore, we detected very small deviations from a planar adsorption geometry, like the bending and tilting of pentacene on $\mathrm{Cu}(111)$. The offset that depends on the tip termination, the macroscopic tip shape, substrate material and applied bias has a larger systematical error. By comparison with DFT and XSW data we find for our Xe terminated tips that this offset is approximately $0.4 \AA$ with an 
error of $0.2 \AA$. Including this tip dependent offset facilitates the determination of absolute adsorption heights of individual molecules by AFM. This detection of the molecular adsorption geometry in combination with the knowledge about the adsorption site provides a detailed picture of the molecular adsorption characteristics.

We thank R. Allenspach and W. Steurer for comments and acknowledge financial support from the EU project ARTIST (contract no. 243421) and the ERC Advanced Grant CEMAS. AT and WL acknowledge support from the ERC Starting Grant VDW-CMAT.

* bsc@zurich.ibm.com

[1] Y. Sugimoto, P. Pou, M. Abe, P. Jelinek, R. Pérez, S. Morita, and O. Custance, Nature 446, 64 (2007).

[2] L. Gross, F. Mohn, N. Moll, P. Liljeroth, and G. Meyer, Science 325, 1110 (2009).

[3] N. Moll, L. Gross, F. Mohn, A. Curioni, and G. Meyer, New J. Phys. 12, 125020 (2010).

[4] F. Mohn, B. Schuler, L. Gross, and G. Meyer, Appl. Phys. Lett. 102, 073109 (2013).

[5] N. Pavliček, B. Fleury, M. Neu, J. Niedenführ, C. Herranz-Lancho, M. Ruben, and J. Repp, Phys. Rev. Lett. 108, 086101 (2012).

[6] S. Henze, O. Bauer, T.-L. Lee, M. Sokolowski, and F. Tautz, Surf. Sci. 601, 1566 (2007).

[7] J. Zegenhagen, Surf. Sci. Rep. 18, 202 (1993).

[8] D. P. Woodruff, Prog. Surf. Sci. 57, 1 (1998).

[9] P. S. Weiss and D. M. Eigler, Phys. Rev. Lett. 71, 3139 (1993).

[10] J. Repp, G. Meyer, K.-H. Rieder, and P. Hyldgaard, Phys. Rev. Lett. 91, 206102 (2003).

[11] J. Lagoute, K. Kanisawa, and S. Fölsch, Phys. Rev. B 70, 245415 (2004).

[12] D. Wegner, R. Yamachika, X. Zhang, Y. Wang, M. F. Crommie, and N. Lorente, Nano Lett. (2013).

[13] G. Teobaldi, K. Lämmle, T. Trevethan, M. Watkins, A. Schwarz, R. Wiesendanger, and A. L. Shluger, Phys. Rev. Lett. 106, 216102 (2011).

[14] L. Gross, F. Mohn, N. Moll, G. Meyer, R. Ebel, W. M.
Abdel-Mageed, and M. Jaspars, Nature Chem. 2, 821 (2010).

[15] F. J. Giessibl, Appl. Phys. Lett. 73, 3956 (1998).

[16] T. R. Albrecht, P. Grutter, D. Horne, and D. Rugar, J. Appl. Phys. 69, 668 (1991).

[17] See supplemental material for a list of all measured and calculated adsorpion heights and details regarding tip preparation, data acquisition and analysis.

[18] The $\mathrm{Cu}$ background spectrum has been subtracted from all $\Delta f(z)$ curves.

[19] F. Mohn, L. Gross, and G. Meyer, Appl. Phys. Lett. 99, 053106 (2011).

[20] F. Mohn, L. Gross, N. Moll, and G. Meyer, Nature Nanotech. 08, 1 (2012).

[21] A. Tkatchenko, L. Romaner, O. Hofmann, E. Zojer, C. Ambrosch-Draxl, and M. Scheffler, MRS Bull. 35, 435 (2010).

[22] V. G. Ruiz, W. Liu, E. Zojer, M. Scheffler, and A. Tkatchenko, Phys. Rev. Lett. 108, 146103 (2012).

[23] A. Tkatchenko and M. Scheffler, Phys. Rev. Lett. 102, 073005 (2009).

[24] C. Bürker, N. Ferri, A. Tkatchenko, A. Gerlach, J. Niederhausen, T. Hosokai, S. Duhm, J. Zegenhagen, N. Koch, and F. Schreiber, Phys. Rev. B 87, 165443 (2013).

[25] W. Liu, J. Carrasco, B. Santra, A. Michaelides, M. Scheffler, and A. Tkatchenko, Phys. Rev. B 86, 245405 (2012).

[26] J. Perdew, K. Burke, and M. Ernzerhof, Phys. Rev. Lett. 77, 3865 (1996).

[27] N. Koch, A. Gerlach, S. Duhm, H. Glowatzki, G. Heimel, A. Vollmer, Y. Sakamoto, T. Suzuki, J. Zegenhagen, J. P. Rabe, and F. Schreiber, J. Am. Chem. Soc. 130, 7300 (2008).

[28] L. Gross, F. Mohn, N. Moll, B. Schuler, A. Criado, E. Guitián, D. Peña, A. Gourdon, and G. Meyer, Science 337, 1326 (2012).

[29] J. Welker and F. J. Giessibl, Science 336, 444 (2012).

[30] A. Zhao, Q. Li, L. Chen, H. Xiang, W. Wang, S. Pan, B. Wang, X. Xiao, J. Yang, J. G. Hou, and Q. Zhu, Science 309, 1542 (2005).

[31] H. Yildirim and A. Kara, J. Phys. Chem. C 117, 2893 (2013).

[32] W. Liu, B. Schuler, N. Moll, L. Gross, and A. Tkatchenko, (to be published) (2013).

[33] N. Moll, L. Gross, F. Mohn, A. Curioni, and G. Meyer, New J. Phys. 14, 083023 (2012). 